\title{
Signal transducer and activator of transcription 3 activation is associated with bladder cancer cell growth and survival Chun-Liang Chen ${ }^{1,2}$, Ling Cen ${ }^{3}$, Jennifer Kohout ${ }^{4}$, Brian Hutzen ${ }^{5}$, Christina Chan ${ }^{2,2}$, Fu-Chuan Hsieh 1,2, Abbey Loy, Victor Huang1,2, Gong Cheng ${ }^{1,2}$ and Jiayuh Lin*1,2,3,4,5
}

\begin{abstract}
Address: ${ }^{1}$ Center for Childhood Cancer, The Research Institute at Nationwide Children's Hospital, Columbus, OH 43205, USA, ${ }^{2}$ Department of Pediatrics, The Ohio State University, Columbus, OH 43205, USA, ${ }^{3}$ Biochmietry Program, The Ohio State University, Columbus, OH 43205, USA, ${ }^{4}$ Integrated Biomedical Science Graduate Program, The Ohio State University, Columbus, OH 43205, USA and ${ }^{5}$ Molecular, Cellular and Developmental Biology Program, The Ohio State University, Columbus, OH 43205, USA

Email: Chun-Liang Chen - Chun-Liang.Chen@nationwidechildrens.org; Ling Cen - Cen.Ling@mayo.edu; Jennifer Kohout - jennifer_kohout@sbcglobal.net; Brian Hutzen - hutzen.1@osu.edu; Christina Chan - chan.254@gmail.com; FuChuan Hsieh - Fu-Chuan.hsieh@nationwidechildrens.org; Abbey Loy - loya@pediatrics.ohio-state.edu; Victor Huang - huang.542@osu.edu; Gong Cheng -cg3468@yahoo.com; Jiayuh Lin* - lin.674@osu.edu

* Corresponding author
\end{abstract}

Published: 21 October 2008

Molecular Cancer 2008, 7:78
Received: 25 September 2007

Accepted: 21 October 2008

isticle is available from: http://www.molecular-cancer.com/content/7///78

(C) 2008 Chen et al; licensee BioMed Central Ltd.

This is an Open Access article distributed under the terms of the Creative Commons Attribution License (http://creativecommons.org/licenses/by/2.0), which permits unrestricted use, distribution, and reproduction in any medium, provided the original work is properly cited.

\begin{abstract}
Background: Constitutive activation of signal transducer and activator of transcription 3 (Stat3) signaling pathway plays an important role in several human cancers. Activation of Stat 3 is dependent on the phosphorylation at the tyrosine residue 705 by upstream kinases and subsequent nuclear translocation after dimerization. It remains unclear whether oncogenic Stat3 signaling pathway is involved in the oncogenesis of bladder cancer.
\end{abstract}

Results: We found that elevated Stat3 phosphorylation in 19 of 100 (19\%) bladder cancer tissues as well as bladder cancer cell lines, WH, UMUC-3 and 253j. To explore whether Stat3 activation is associated with cell growth and survival of bladder cancer, we targeted the Stat 3 signaling pathway in bladder cancer cells using an adenovirus-mediated dominant-negative Stat3 (Y705F) and a small molecule compound, STA-2I. Both prohibited cell growth and induction of apoptosis in these bladder cancer cell lines but not in normal bladder smooth muscle cell (BdSMC). The survival inhibition might be mediated through apoptotic caspase 3, 8 and 9 pathways. Moreover, downregulation of anti-apoptotic genes (Bcl-2, Bcl-xL and survivin) and a cell cycle regulating gene (cyclin DI) was associated with the cell growth inhibition and apoptosis.

Conclusion: These results indicated that activation of Stat3 is crucial for bladder cancer cell growth and survival. Therefore, interference of Stat3 signaling pathway emerges as a potential therapeutic approach for bladder cancer.

\section{Background}

Several malignancies have been shown to result from constitutive activation of STATs, in particular Stat 3 and 5
$[1,2]$. Stat 3 is widely expressed in normal tissues and transiently activated and then inactivated by a group of signaling proteins, such as SH2-containing tyrosine 
phosphotases (SHP1 and SHP2), protein inhibitors of activated STATs (PIAS) and suppressor of cytokine signaling proteins/extracellular signaling regulated kinase (SOCS/ERK) cascades [3-5]. In a variety of human cancers, defects in these signaling pathways or persistent presence of up-stream activators would lead to constitutive activation of Stat 3 and tumorgenesis [6,7]. Interference of constitutive Stat3 signaling pathway suppresses chemotherapy resistance, tumor growth and metastasis, induces cancer cell death and therefore shows great potential for cancer therapy $[8,9]$.

Several lines of evidence suggest that constitutive activation of Stat 3 might play a role in bladder malignancy. Bladder cancer is one of the common malignancies and molecular causes for its progress and development have been intensively investigated [10-12]. However, the detailed picture of oncogenic pathways for bladder cancer has just begun to be revealed [11]. Bladder cancer is induced by amplification of oncogenes [eg. fibroblast growth factor receptor 3 (FGFR3) and Ras gene] or by mutational defects in tumor suppressor genes (eg. PTCH \& PTEN). These diverse genetic changes lead to oncogenic signalings via MAPK, PI-3 kinase, AKT and c-Myc pathways. Overactive FGFR3 and ERBB2 in bladder cancer presumably would activate Stat 3 that is down-stream to these two receptor tyrosine kinases [10]. Another line of evidence is that overexpression of Stat3-regulated anti-apoptotic genes (Bcl-2, Bcl-xL and survivin) is found in bladder cancer. Overexpression of these genes renders bladder cancer progression, accelerated rates of recurrences, antiapoptosis and chemotherapeutic resistance [13-18]. The role of activated Stat3 in bladder cancer remained speculative until the recent report showed that Stat 3 activation correlated with malignant characteristics of T24 bladder cancer cells [19]. This implicates that activation of Stat3 may play a role in the development of bladder cancer.

We initiated a study to explore any further relation between activation of Stat 3 and bladder malignancy. We found that 19 of 100 (19\%) bladder cancer biopsy tissues had elevated expression of phosphorylated-Stat3 (pStat3) using an immunohistochemical staining with a pStat3 specific monoclonal antibody. In addition, elevated p-Stat 3 expression was also found in bladder cancer cell lines, UMUC-3, 253J and WH. Thereafter, we targeted the activated Stat3 signal pathway using a dominant negative Stat3 Y705F (dnStat3) and a small molecule inhibitor, STA-21 $[8,20]$. Inhibition of Stat3 pathway suppressed cell growth of bladder cancer cells in vitro. DnStat3 and STA-21 also induced apoptosis as revealed by immunostaining of cleaved caspases 3, 8 and 9 in bladder cancer cells. Down regulation of anti-apoptotic genes (Bcl-2, Bcl$\mathrm{xL}$ and survivin) and a cell-cycle regulating gene, cyclin D1, were correlated with dnStat3- and STA-21 induced apoptosis and cell growth inhibition. Taken together, Stat3 activation may play a pivotal role in bladder cancer cell growth and survival and serve as a novel therapeutic target for this type of cancer.

\section{Results \\ p-Stat3 was elevated in bladder cancer tissues}

Tissue microarray immunohistochemistry indicated that Stat3 phosphorylation was elevated in bladder cancer tissues. Three representative bladder cancer tissues with pStat3 positive immunostaining (scale 2-3) are shown (Figure 1B-D), whereas normal bladder tissues were negative or very weak (scale $0-1$ ) with immunostaining (Figure $1 \mathrm{~A}$ ). The elevated $\mathrm{p}$-Stat 3 in the bladder cancer tissues was scored and summarized according to immunostaining intensities. Two of the bladder cancer tissues were not included for immunostaining scoring because no staging information is available. Nineteen out of 100 bladder cancer tissues were positive for p-Stat 3 immunostaining (scale 2-3).

The clinicopathological data for 102 bladder cancer tissues are classified in Table 1 . The samples represented 76 male and 26 female patients, with the majority of patients (95.1\%, 97 out of 102) between 41-90 years old. Fortyfive tissues and 57 tissues were staged and graded, respectively. Forty of 45 staged tissues $(89 \%)$ had no signs of regional lymph nodes nor distant organ metastases. According to histological features, the bladder cancer tissues were identified as urothelial carcinoma $(85 \%)$, squamous cell carcinoma (3\%), adenocarcinoma (2\%) and mixed carcinomas $(10 \%)$.

\section{p-Stat3 was also elevated in bladder cancer cell lines}

Western blot analysis showed that elevated p-Stat 3 was also found in bladder cancer cell lines, UMUC-3, 253J and WH (Figure 2). Very low or no p-Stat 3 was detected in the same amount of T24 and TCC cell lysates as revealed by the internal controls of GAPDH. All bladder cancer cell lines examined have very similar total Stat 3 expression levels.

\section{rAd-mediated transduction of dnStat3 in bladder cancer cell lines}

One way to investigate the functions of activation of Stat3 in bladder cancer is to interrupt Stat 3 signaling pathway in bladder cancer cell lines with elevated p-Stat3. To that end, WH and UMUC-3 were transduced with rAd/ $\mathrm{dnStat} 3$. The two cell lines were infected with $\mathrm{rAd} / \mathrm{dnStat} 3$ ( $\mathrm{moi}=100,250$, and 500). The expression of dnStat 3 in WH cells was observed and shown at 48 hours post infection (Figure 3). FLAG-tagged dnStat 3 expressions in $10 \mu \mathrm{g}$ of bladder cancer cell lysates were detected by western blots using an anti-FLAG antibody. Total Stat3 expressions correspondingly reflected the dose-dependent 

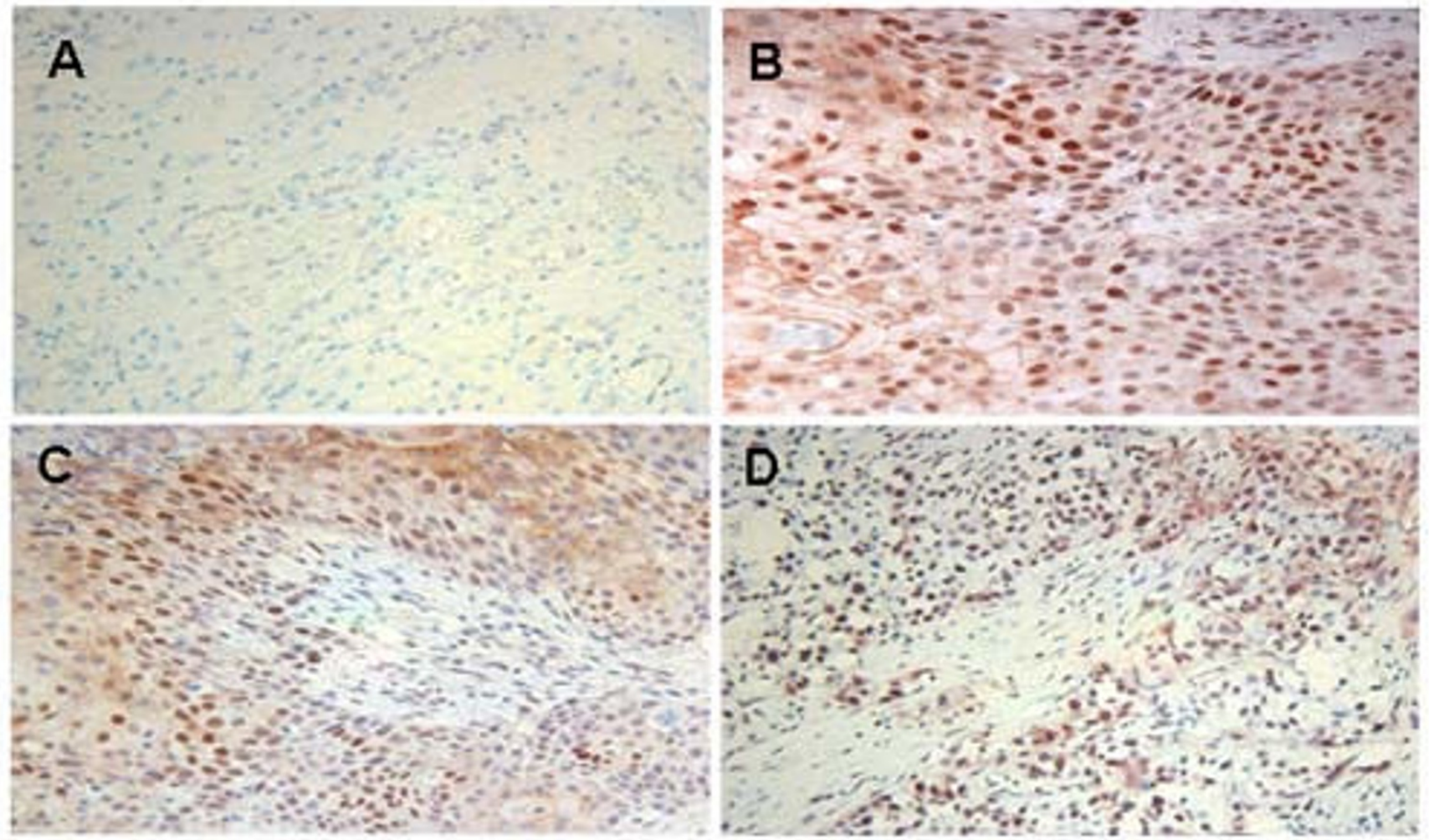

\section{Figure I}

p-Stat3 was elevated in bladder cancer tissues. (A) normal tissue, (B) squamous cell carcinoma (Stage II), (C) urothelial carcinoma (stage III), (D) urothelial carcinoma (stage IV). Normal tissues appeared negative in p-Stat3 staining. The nuclei were counterstained with hematoxylin blue. Image magnification was I00x.

increase of dnStat3 expressions in these bladder cancer cells.

\section{Targeting Stat3 signaling pathway using dnStat3 and STA- $2 I$ induced cell growth and viability inhibition in bladder cancer cells}

Bladder cancer cell growth was significantly suppressed in the presence of dnStat3 and STA-21. UMUC-3 and WH cells were transduced with either $\mathrm{rAd} / \mathrm{dnStat} 3$ or $\mathrm{rAd} /$ eGFP (moi $=100$ and 500). Cell growth is presented in cell densities normalized to untransduced controls at day 2 and day 4 post-infection (Figure 4A and 4B). The growth rates of untransduced cells were set at $100 \%$. There was $20-30 \%$ reduction in UMUC-3 cell growth at day 4 postinfection with rAd/eGFP; more notably, the UMUC-3 cell line transduced with $\mathrm{rAd} / \mathrm{dnStat} 3$ ( $\mathrm{moi}=100$ and 500) was reduced down to $11.9 \pm 0.3 \%(\mathrm{P}<0.05)$ and $1.3 \pm$ $0.3 \%(\mathrm{P}<0.005)$ of cell growth at day 4 compared to untransduced controls (Figure 4A). The discrepancies between cell growth of bladder cancer cells transduced with rAd/eGFP and rAd/dnStat3 apparently reflects dnStat3-specific inhibitory effects. The dnStat3-mediated inhibition of cell growth was even more dramatic as observed in WH cells (Figure 4B). WH cells transduced with $\mathrm{rAd} / \mathrm{dnStat} 3$ ( $\mathrm{moi}=100$ and 500 ) had only $50.2 \pm$ $13.5 \%(P<0.05)$ and $16.1 \pm 2.2 \%(P<0.005)$ of cell growth compared to untransduced controls at day 2 postinfection; evenmore, at day 4 post-infection, cell growth of $\mathrm{rAd} / \mathrm{dnSTat3-transduced} \mathrm{WH}$ cells was decreased to only $1.1 \%$ and $0.5 \%$ of untransduced controls. After 4 day of infection with a higher dose of $\mathrm{rAd} / \mathrm{Stat} 3(\mathrm{moi}=1000)$, a cell viability assay (MTT) also revealed that WH and UMUC with higher content of p-Stat3 only maintained $34.8 \pm 1.4 \%$ and $51.9 \pm 8.5 \%$ cell viability of untreated controls (Figure 4C). On the contrary, TCC and T24 with much lower or undetectable p-Stat 3 contents had much higher cell viability with $90.5 \pm 3.6 \%$ and $73.6 \pm 7.5 \%$ of untreated controls under the same experimental conditions. The overall cell viability of cells treated with $\mathrm{rAd} /$ dnStat 3 was decreased along the time, while that of cells untransduced or treated with $\mathrm{rAd} / \mathrm{eGFP}$ was increased (data not shown).

As MTT assay showed, similar inhibition on cell growth and viability was observed in bladder cancer cells treated with $30 \mu \mathrm{M}$ STA-21 (Figure 4C). Viability of bladder can- 
Table I: Clinicopathological parameters of urinary bladder cancers used

\begin{tabular}{|c|c|c|}
\hline Clinicopathological parameters & & Numbers (\%) \\
\hline \multirow[t]{2}{*}{ Gender } & Male & $76(75.9)$ \\
\hline & Female & $26(24.1)$ \\
\hline \multirow[t]{6}{*}{ Age (years) } & $20-40$ & $4(3.9)$ \\
\hline & $41-60$ & $41(40.2)$ \\
\hline & $61-90$ & $56(54.9)$ \\
\hline & $N / A^{a}$ & $\mathrm{I}(\mathrm{I} .0)$ \\
\hline & Mean & 62 \\
\hline & Median & 64 \\
\hline \multirow[t]{7}{*}{ Stage (total 45) } & $0 a^{b}$ & $6(13.3)$ \\
\hline & Oisc & $3(6.7)$ \\
\hline & 1 & $16(35.6)$ \\
\hline & II & $7(15.6)$ \\
\hline & III & $8(17.8)$ \\
\hline & IV & $3(6.7)$ \\
\hline & $\mathrm{N} / \mathrm{A}$ & $2(4.4)$ \\
\hline \multirow[t]{3}{*}{ Grade (Total 57) } & 1 & $5(8.8)$ \\
\hline & ॥ & $17(29.8)$ \\
\hline & III & $35(61.4)$ \\
\hline Regional lymph node and/or distant metastasis & NOMOd & $40(88.9)$ \\
\hline \multirow[t]{4}{*}{ (total 45) } & NIMOe & I (2.2) \\
\hline & NOMIf & $\mathrm{I}(2.2)$ \\
\hline & NIMIg & I (2.2) \\
\hline & N/A & $2(4.4)$ \\
\hline \multirow[t]{4}{*}{ Histology (total 54) } & Urothelial carcinoma & $87(85.3)$ \\
\hline & Squamous cell carcinoma & $3(2.9)$ \\
\hline & Adenocarcinoma & $2(2.0)$ \\
\hline & Mixed carcinomas & $10(9.8)$ \\
\hline
\end{tabular}

a N/A - Information are not available

b $\mathrm{Oa}$ - Cancer is noninvasive

c Ois - Noninvasive, flat carcinoma in situ

d NOMO - No regional lymph nodes neither distant organs metastasis

e NIMO - Metastasis in I to 3 regional lymph nodes

${ }^{f} \mathrm{NOMI}$ - No regional lymph nodes but distant organs metastasis

$\mathrm{gNIMI}$ - Metastasis both in regional lymph nodes and distant organs

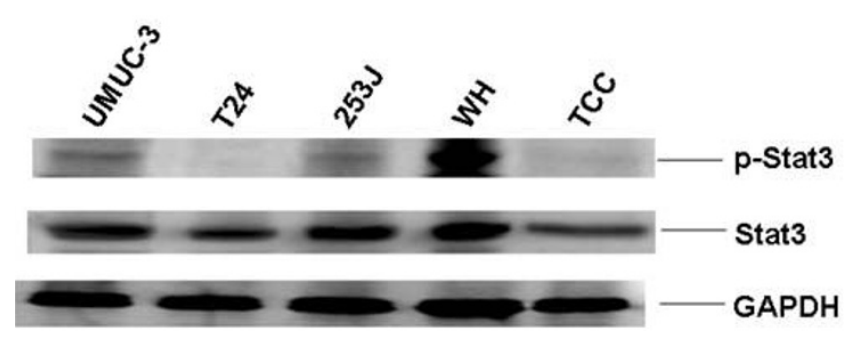

Figure 2

Elevated p-Stat3 (Y705) was found in bladder cancer cell lines. One hundred $\mu \mathrm{g}$ of cell lysates from bladder cancer cells were subject to western blot analysis using anti-pStat3 (Y705), -Stat3 and -GAPDH specific antibodies. cer cells and BdSMC treated with DMSO was about the same as untreated controls. However, exposure to STA-21 greatly reduced cell viability of 253J, UMUC3 and WH $(38.1 \pm 0.74 \%, 11.4 \pm 1.5 \%$, and $29.0 \pm 6.7 \%)$. Interestingly, STA-21 had very minimal effects on BdSMC cell viability $(91.3 \pm 4.4 \%)$. This indicated that STA-21 inhibition is specific to bladder cancer cells that have constitutive activation of Stat3. In addition, the decreased overall viability of cells treated with STA-21 was consistent with that observed in cells treated with $\mathrm{rAd} / \mathrm{dnStat} 3$. These together suggested that bladder cancer cell death associated with inhibition of Stat3 pathway might have occurred. 


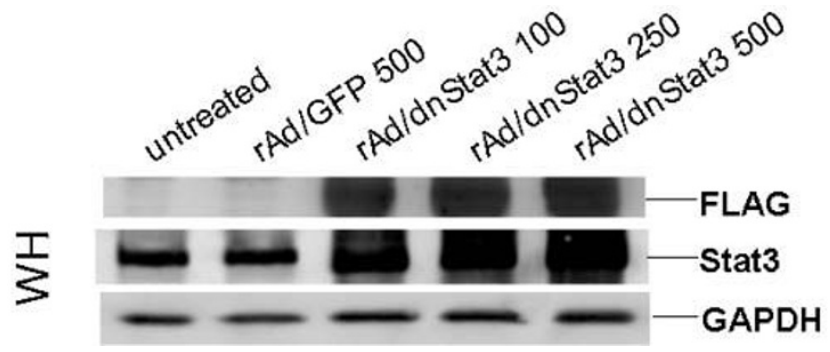

Figure 3

Transduction of dnStat3 (Y705F) in bladder cancer cell lines using a recombinant adenoviral vector. $A$ representative bladder cancer cell line, $\mathrm{WH}$, was transduced with designated mois of $\mathrm{rAd} / \mathrm{dnStat} 3$ and $\mathrm{rAd} / \mathrm{eGFP}$. Twenty $\mu \mathrm{g}$ of cell proteins were fractionated on I2\% PAGE gels and immunoblotted with specific antibodies against FLAG, Stat3 and GAPDH.

\section{Inhibition of Stat3 pathway induces activation of apoptotic caspase pathways}

Transduction of dnStat 3 in bladder cancer cells induced activation of apoptotic caspases 3, 8, and 9 in those cells transduced with rAd/dnStat3. UMUC-3 and WH were fixed at day 2 and day 1, respectively, post-transduction of $\mathrm{rAd} / \mathrm{eGFP}$ or $\mathrm{rAd} / \mathrm{dnStat} 3$ and then subject to immunofluorescent staining using antibodies that recognize cleaved caspases 3, 8 and 9 for apoptosis evaluations. Only rare sporadic cells were found stained by anticleaved caspases 3, 8, and 9 antibodies in negative UMUC-3 and WH controls (untransduced or transduced with rAd/eGFP) (Figure 5A \&5B). However, many dnStat3-transduced cells were positive in anti-cleaved caspases 3 (44.2 and 53\%), 8 (53.5 and 74.4\%), and 9 (74.2 and $42.4 \%$ ) immunostaining (Figure 5B).

Targeting Stat3 pathway using STA-21 also led to increased cleavage of caspase 3 after 72 hours of treatment in 253J, UMUC-3 and WH (Figure 5C and data not shown). About $6.4-13 \%$ cells were cleaved caspase 3 positive in these three STA-21-treated bladder cancer cell lines, compared to less than $0.5 \%$ in untreated or DMSOtreated cells. STAT- 21 treatment did not seem to induce caspase 3 cleavage in BdSMC cells with less than $0.1 \%$ appearing positive with anti cleaved caspase 3 immunoreactivity. The increased apoptotic caspase activation implicated that apoptosis could be one of mechanisms underlying the decreased cell viability in bladder cancer cells in that Stat 3 pathway was compromised by treatment of rAd/Stat3 or STA-21.

\section{dnStat3 down-regulated anti-apoptotic genes and cyclin $D I$ in bladder cancer cells}

We then would like to explore possible mechanisms for dnStat3-induced cell growth inhibition and activation of apoptotic caspases in bladder cancer cells. It is likely that inhibition of Stat 3 pathway by dnStat3 or STA-21 down regulated $\mathrm{Bcl}-2, \mathrm{Bcl}-\mathrm{xL}$ and survivin as well as cell cycle regulating gene, cyclin D1. Reduced expressions of these genes may contribute to bladder cancer cell growth inhibition and apoptotic caspase activation. UMUC-3 and WH cells were transduced with either rAd/eGFP or rAd/ dnStat 3 (moi = 1000 and 500) for 27 hours and 48 hours, respectively. Expression of survivin, Mcl-1, Bcl-2, Bcl-xL and cyclin D1 proteins in these cells were evaluated using western blot analysis and densitometric quantification. In UMUC-3 cells, Bcl-2 (47\% untreated control), Bcl-xL (55.4\%) and survivin (9.7\%) were down regulated by the transduction of dnStat 3 as compared to expressions in untransduced cells or cells transduced with rAd/eGFP (Figure 6A), whereas Mcl-1 (100\%) remained intact. Expression of the three genes in rAd/eGFP-transduced cells ( $\mathrm{moi}=1000$ ) was only slightly changed as compared to the untransduced cells. survivin(67.1\% untreated control), Bcl-xL (61.1\%) and cyclin -D1 (62.7\%) expression was detected to be decreased when the same cell line treated with $30 \mu \mathrm{M}$ STA-21 for 4 days (Figure 6C).

We also examined the expression of anti-apoptotic genes and cyclin D1 in WH bladder cancer cells when Stat3 pathway was targeted by $\mathrm{rAd} / \mathrm{dnStat} 3$ or STA-21. Mcl-1 (80\% untreated control) was apparently not affected as compared with the control treated with rAd/eGFP but Bcl$2(16.6 \%)$ and Bcl-xL (46.9\%) protein expressions were down regulated by the transduction of dnStat3 (Figure $6 \mathrm{~B})$. In addition, cyclin D1 expression (1.9\% untreated control) was almost completely inhibited by the interference of Stat3 signaling pathway. Expression reduction in two anti-apoptosis genes (Bcl-2 and Bcl-xL) and cyclin D1 were consistent with apoptosis and cell growth inhibition in WH cells. Four days of treatment of STA-21 showed similar but less reduction in survivin (19.6\% untreated control), Bcl-2 (64\%) and Bcl-xL (79.2\%) expressions.

\section{Discussion}

Constitutive activation of Stat3 signaling pathway is frequently detected in several types of human cancers. This report was to explore the correlation between bladder cancer and Stat 3 status in bladder cancer tissues and cell lines. We found that elevated p-Stat 3 expression is found in both bladder cancer tissues and cell lines. Among 100 primary bladder cancer biopsy tissues, $19 \%$ appears positive in p-Stat3 immunostaining in nuclei, cytoplasm or both compartments. Majorities of bladder cancer tissues examined are negative for $\mathrm{p}$-Stat 3 and may result from other causes for this kind of cancer [10]. Elevated p-Stat 3 expression is also found in bladder cancer cells, UMUC-3, WH and T24. These suggest that elevated p-Stat 3 might contribute to some of bladder malignancy. Phosphorylation at tyrosine 705 is required for the activation of Stat3. Ele- 
A.

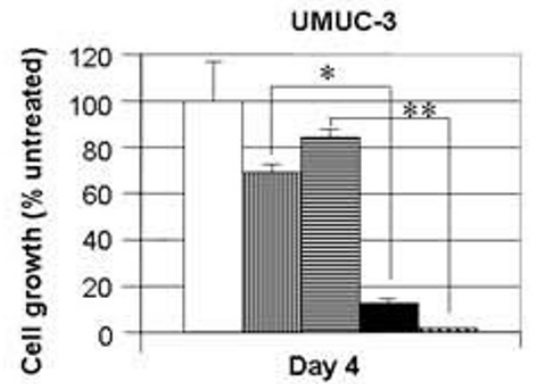

B.

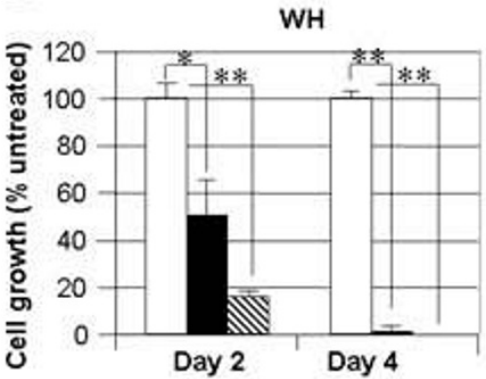

C.

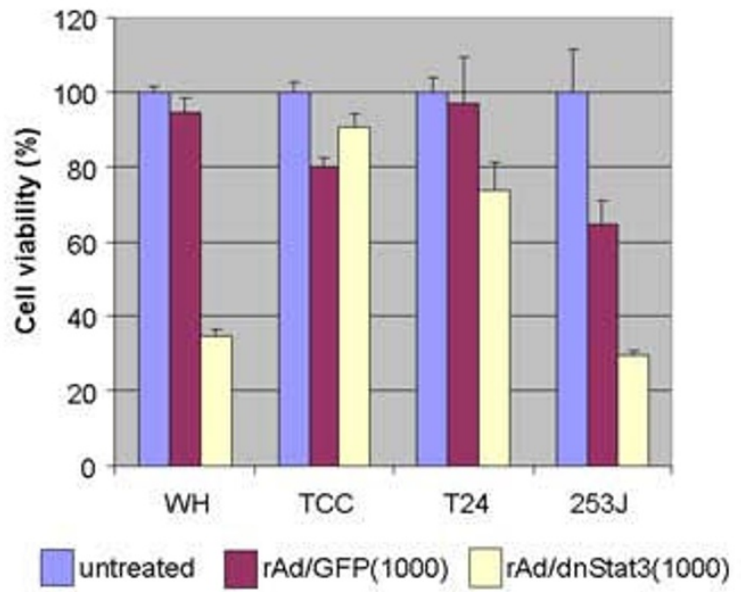

D.

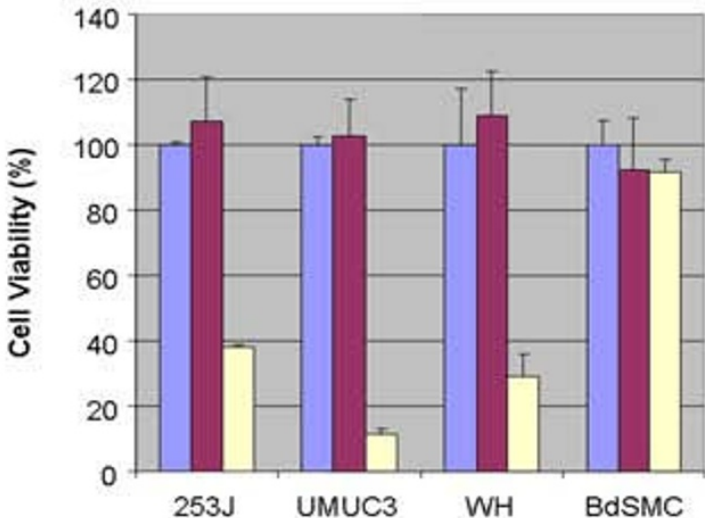

Untreated DMSO $\square$ 30uM STA21

Figure 4 (see legend on next page) 
Figure 4 (see previous page)

Targeting Stat3 pathway inhibits cell growth and viability. Transduction of dnStat3 suppressed cell growth of bladder cancer cell lines, (A) UMUC-3, at day 4 post infection and (B) WH, at day 2 and 4 post infection. Growth of bladder cancer cells was strongly suppressed by the expression of dnStat3. Cells were transduced with either $\mathrm{rAd} / \mathrm{dnStat} 3 \mathrm{or} \mathrm{rAd} / \mathrm{eGFP}$ (moi $=100$ or 500) while untransduced cells served as negative controls. Cells in five random individual microscopic fields (I00x) were scored at day 2 or day 4 post-infection. (C) MTT assay also shows that transduction of dnStat3 limits cell growth of bladder cancer cell lines (WH and 253J) with higher content of p-Stat3 but not that of those cells with less p-Stat3 at 4 day postinfection. Cell growth was shown in cell density over control cell density (\%). (D) Cell viability of cancer cells was greatly reduced after treatment of STA-2 I, a Stat3 dimerization inhibitor. Cells were treated with DMSO or $30 \mu$ M STA-2I for 4 days. STA-2I had very limited effects on BdSMC cell viability. Cell viability was determined using a MTT assay. Averages and standard deviations for data points were derived from triplicate experiments. $*(P<0.05)$ and $* *(P<0.005)$ indicate paired t-test statistic significance.

vated Stat 3 phosphorylation in these bladder tissues and cell lines might result from abnormal overactive upstream oncogenic FGFR or ERBB2 in these cancer tissues [10,21]. A recent study shows that overactive Stat 3 serves as the signal mediator between EGF and MMP-1 for bladder cancer cell migration, invasion and tumor formation [19]. Alternative explanation is the down regulation of counter balancing signal transduction pathways, such as SH2containing tyrosine phosphotase (SHP1 and 2), protein inhibitors of activated Stats (PIAS), and suppressors of cytokine signaling (SOCS), could also contribute to higher Stat3 phosphorylation in these bladder cancer tissues or cell lines [5]. These need further verifications using tissue microarray immunohistochemistry or quantitative PCR.

Our data suggest that bladder cancer cells might utilize Stat3 signaling pathway for cell growth and survival. Interruption of Stat 3 pathway using a dnStat 3 or STA-21 affects bladder cancer cell growth and induces the activation of apoptotic caspases. DnStat3 may inhibit phosphorylation and dimerization of endogenous Stat3 [22-24] and down regulates a group of survival and proliferation genes [2527]. STA-21 was discovered from a virtual drug screen and showed efficacy in blocking Stat3 dimerization and translocation into nuclear compartments [8]. Our data, consistent with previous studies, has delineated part of the relationship between elevated p-Stat3 expression and bladder cancer [19], although mechanisms for cell growth inhibition and cell death by dnStat 3 in bladder cancer cell UMUC-3 and WH remain largely unclear. Reduction of cyclin-D1 expression in WH and UMUC-3 cells might be part of the causes for cell growth inhibition. This is consistent with previous study that targeting Stat3 signaling with dnStat 3 suppresses cell-cycle-related genes, including cyclin-D1, in ALK-positive anaplastic large cell lymphoma [28].

Interruption of Stat3 pathway by dnStat3 and STA-21 leads to activation of caspase 3 signaling in bladder cancer cells. Apparently, dnStat3-induced cleavage of caspase 3 is also mediated through caspases 8 and 9 pathways. Caspases 8 and 9 are key initiator caspases for two largely independent apoptotic pathways mediated by death receptors and stresses [29-32]. Cleaved caspase 8 suggests an autocrine signal(s) following dnStat 3 transduction in bladder cancer cells. Fas, TRAIL receptors and their ligands are usually suppressed in several cancers to prevent apoptosis $[33,34]$. Stat3 has been shown to directly down regulate Fas, TRAIL, and TGF- $\alpha$ [35-37]. To Target Stat3 signaling pathway using Stat $3 \beta$ upregulates TRAIL and a secretory apoptotic signal(s) in B16 tumor cells. What death receptor(s) is involved in dnStat3-induced apoptosis in bladder cancer cells acquires further investigations.

Activation of caspase 9 pathway in bladder cancer cells is very likely triggered by down regulation of $\mathrm{Bcl}-2$ family genes and inhibitors of apoptotic proteins (IAP). We observed that two Bcl-2 family genes (Bcl-2 and $\mathrm{Bcl}-\mathrm{xL}$ ) and an IAP gene (survivin) are negatively affected at protein level by dnStat3 and STA-21. Overexpression of Bcl-2, $\mathrm{Bcl}-\mathrm{xL}$, and survivin in several cancers overcomes severe tumor environments and facilitates cancer progression, chemotherapeutic resistance and higher rate of recurrence $[15,17,18,38,39]$. Down regulation of these genes likely contributes to the dnStat3- and STA-21-induced activation of apoptotic caspases in bladder cancer cells. DnStat3 also inhibits Stat3 signaling in ALK-positive anaplastic large cell lymphoma by suppression of several Bcl-2 family genes [28]. Activated Stat3 promoting cancer survival and proliferation has been demonstrated in several cancers [8,40-48]. To suppress Stat3 signaling pathway using anti-sense RNA, siRNA, small molecules, decoy-oligos and dnStat 3 results in cancer cell growth inhibition and apoptosis. It appears that targeting dnStat 3 signaling pathway could be an effective therapeutic approach for bladder cancer expressing constitutive activation of Stat3.

\section{Conclusion}

Our data show that Stat3 phosphorylation is elevated and may play a pivotal role in cell growth and survival of bladder cancer. Cell growth inhibition and apoptosis can be 
A.

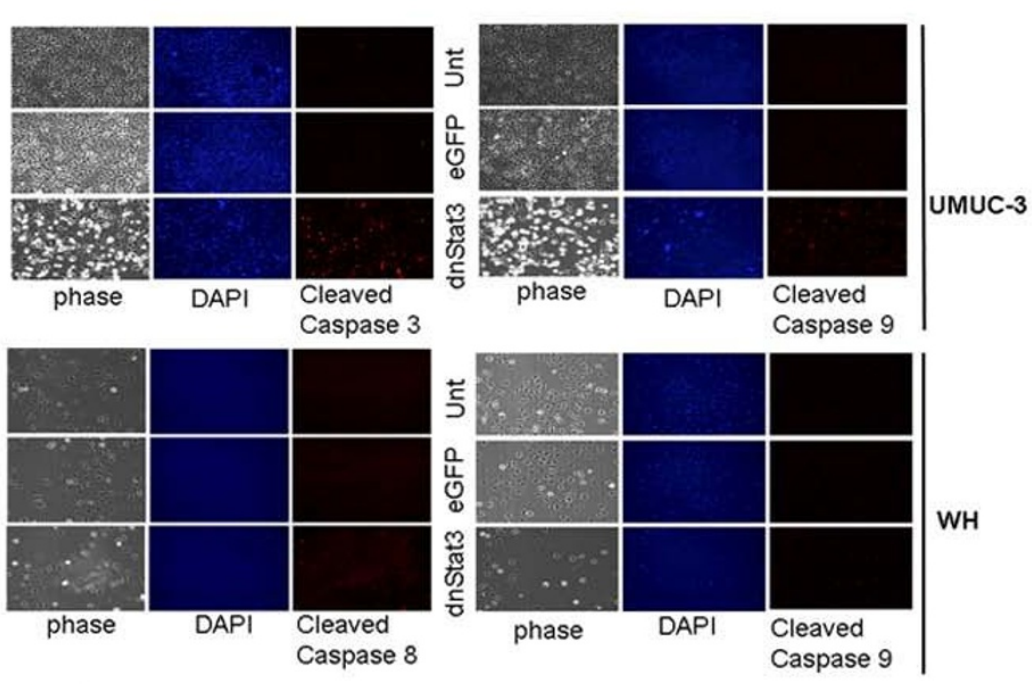

B.

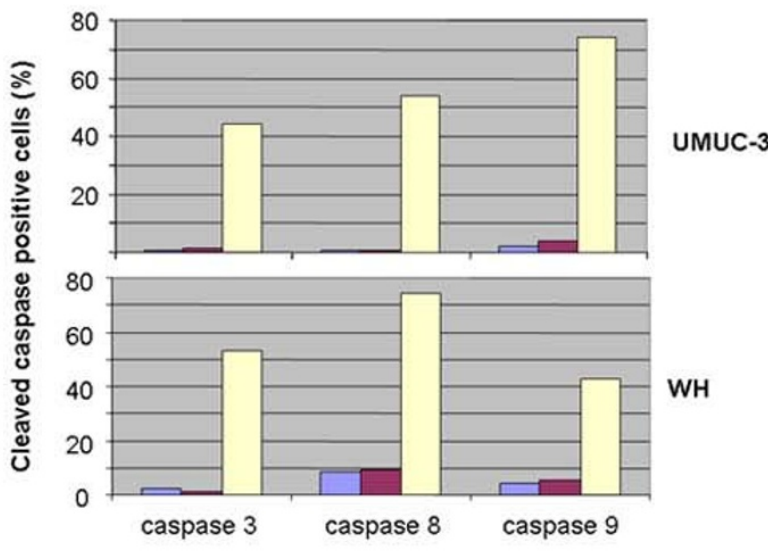

$\square$ Untreated $\square \mathrm{rAd} / \mathrm{GFP} \quad \square \mathrm{rAd} / \mathrm{dnStat3}$

c.
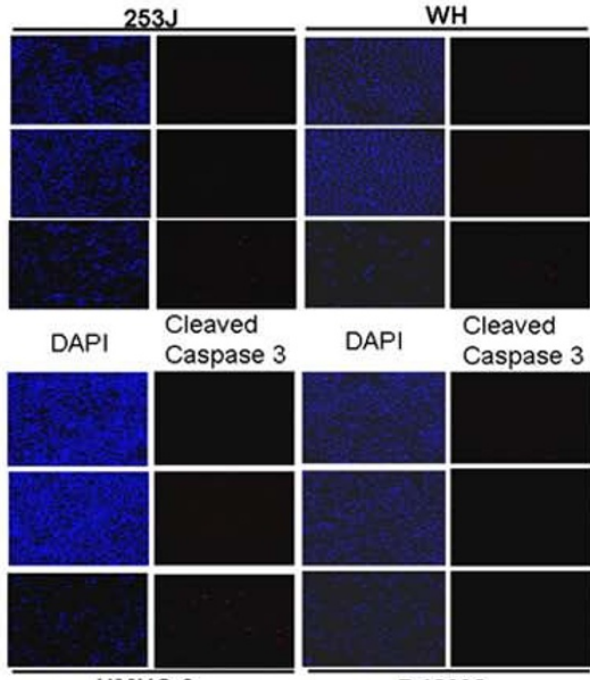

untreated

DMSO

STA-21

UMUC-3

BdSMC

Figure 5 (see legend on next page) 
Figure 5 (see previous page)

Inhibition of Stat3 pathway induces apoptosis through caspase 3, 8 and 9 pathways in bladder cancer cells but not in bladder smooth muscle cells. (A) Cleaved caspase 3, 8 and 9 staining in dnStat3-transduced UMUC-3 and WH at 48 $\mathrm{h}$ post-infection and $3 \mathrm{I} \mathrm{h}$ post-infection, respectively. Cells were transduced, fixed and then immunostained with anti-cleaved caspases 3, 8 and 9 antibodies. Cleaved caspases 3, 8 and 9 immunoreactivies were observed in cells transduced with rAd/ dnStat3 but not in the cells transduced with rAd/eGFP or negative control. Cleaved caspase 3, 8, 9: anti-cleaved-caspases 3, 8, \& 9 antibody immuno-fluorescent staining; Unt: untreated; DAPI: nuclear staining with DAPI; phase:phase-contrast. Magnification of images was $100 \times$. (B) 45-75\% of UMUC-3 and WH cells transduced with rAd/dnStat 3 were cleaved caspase 3, 8, and 9 positive. (C) STA-2I also induced apoptosis (cleaved caspase 3 staining) in 253J, WH and UMUC-3 cells but not in BdSMC cells.

induced in bladder cancer cell lines using either a dnStat3 or a small molecule inhibitor, STA-21 to interfere with the Stat3 signaling pathway. The Stat3 signaling pathway appears as a potential target for bladder cancer therapy.

\section{Methods \\ Cell culture}

Bladder cancer cell lines were purchased from American Type Culture Collection (ATCC). Cell lines were maintained in $1 \times$ DMEM supplemented with $10 \%$ fetal bovine serum and $100 \mathrm{U} / \mathrm{ml}$ penicilin/streptomycin/amphotericin B (Mediatech, Herndon, VA) at $37^{\circ} \mathrm{C}$, aired with $5 \%$ $\mathrm{CO}_{2}$. Bladder smooth muscle cells (BdSMC) were purchased from Cambrex Bio Science and maintained in
$\mathrm{SmGM}^{\oplus}$-2-Smooth muscle medium (Cambrex, Chicago, IL) supplemented with 5\% FBS.

\section{Bladder cancer tissue microarray immunohistochemistry}

Stat3 phosphorylation status in bladder cancer tissues were examined using immunohistochemistry with a pStat3 (Y705)-specific monoclonal antibody (Cell Signaling Tech., Danvers, MA). We stained bladder cancer tissue samples $(\mathrm{n}=102)$ on tissue microarray slides from two different providers (US Biomax, Inc., Rockville, MD and ISU ABXIS Co., Seoul, Korea). The immunohistochemistry and scoring of p-Stat 3 expression were described previously [49]. Most of the p-Stat3 positive cancer tissues showed staining in greater than $50 \%$ of each sample.
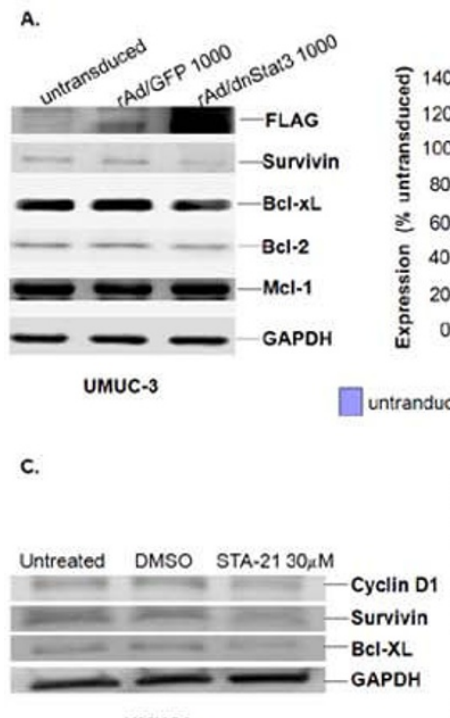

UMUC3

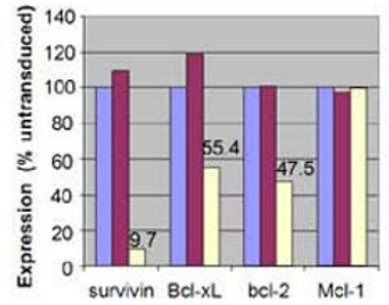

untranduced $\square$ rAd/GFP(1000) $\square \mathrm{rAd} / \mathrm{dnStat3}(1000)$

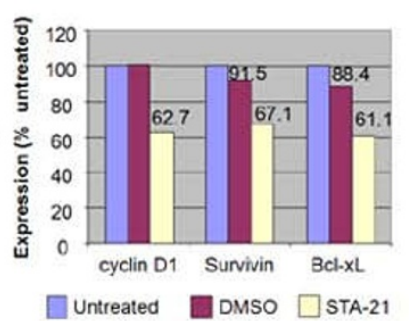

B.
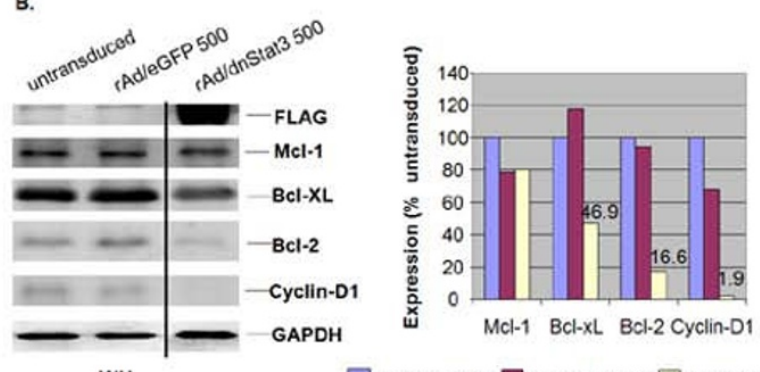

untransduced $\square$ rad/GFP(500) $\square$ rAd/dnStat3(500)
D.

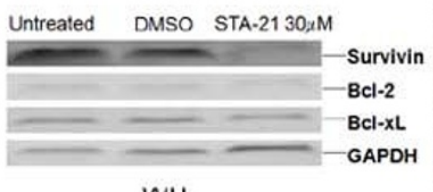

WH

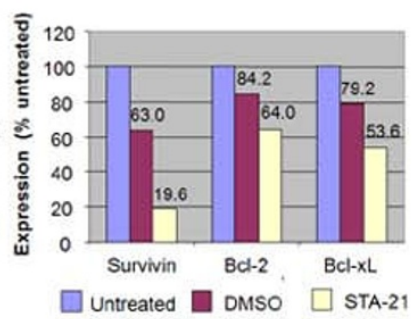

Figure 6

Inhibition of Stat3 pathway by dnStat3 down-regulates survival genes and cyclin-D I gene. The protein expression levels are shown in (A) UMUC-3 at 27 hours and (B) WH at 48 hours post-infection with rAd/dnStat3 or the control vector, rAd/eGFP as well as in (C) UMUC-3 and (D) WH that are treated with designated concentrations of STA-2I for 4 days. Protein expressions of survival genes (Bcl-xL, Bcl-2, $\mathrm{Mcl}-\mathrm{I}$ and survivin) and cyclin-DI were subject to densitometric quantification and shown in percents of the untreated controls after being normalized to the GAPDH expression. A representative experiment of triplicates is shown. 


\section{Western blots}

Western blots were carried out according to protocols described previously [49]. 10 to $100 \mu \mathrm{g}$ of cellular proteins were resolved on $10 \%$ or $14 \%$ SDS-PAGE gels before transfer, immunoblotting, and visualization of specific protein bands. Antibodies were purchased separately and used to recognize FLAG (Sigma, St. Louis, MO) GAPDH (Chemicon International, Temecula, CA). Stat3, p-Stat3 (Y705) (Cell Signaling Tech., Danvers, MA), Bcl-2, Bcl- $\mathrm{x}_{\mathrm{L}}$ (Biosciences, Inc. Franklin Lakes, NJ,), Mcl-1, cyclin D1 (Lab Vision Corp., Fremont, CA) and survivin (UpState, Charlotteville, VG)

For expression comparison, each protein expression was presented in a percentage of its corresponding untreated control after densitometric quantification and normalization to the GAPDH expression. A representative one from duplicated experiments was presented.

\section{Transduction of dnStat3 in bladder cancer cell lines}

The construction of recombinant Adenovirus/CMVdnStat3 Y705F (rAd/dnStat3) is described previously [23]. DnStat 3 was generated from Stat 3 by changing the tyrosine at position 705 into phenylalanine. The dnStat 3 protein product is tagged with a 6 -repeat FLAG sequence for detection and cannot be activated through tyrosine phosphorylation. About $2 \times 10^{5} \mathrm{WH}$ and UMUC-3 cells were transduced with rAd/dnStat3 or rAd/CMV-eGFP (rAd/ eGFP) (Applied Viromics, Fremont, CA) at variant multiplicities of infection (moi) based on TCID50 assay using 293 cells. For cell growth experiments, cell numbers were enumerated at day 2 or 4 post-infection. Cell counts in 5 random fields of view (magnification 100×) were obtained for each treatment and control. Cell growth rates were presented in percentages of cell density of untreated controls and averaged from triplicate experiments.

\section{Treatment of STA-2 I and Cell viability assay}

Approximately 5000 cells were grown in $100 \mu \mathrm{l}$ 10\% FBSsupplemented DMEM medium in 96-well flat-bottomed plates overnight. Cells were exposed to STA-21 $(30 \mu \mathrm{M})$ that was dissolved in dimethyl sulfoxide (DMSO) before added to the medium. Cell viability was analyzed by the MTT [3-(4, 5-dimethyl-2-thiazolyl)-2, 5-diphenyl-2Htetrazolium bromide] (Sigma) assay in three replicates. At the time of assay end-point, cells were treated with MTT (1 $\mathrm{mg} / \mathrm{ml}$ ) for 3-4 hours. Colormetric quntitation was determined by an EL808 Ultra Microplate Reader (Bio-Tek Intruments, Inc) after formazan was dissolved in $25 \% \mathrm{~N}$, $\mathrm{N}$-dimethylformamide and $10 \%$ SDS in a light-proof condition overnight.

\section{Caspases 3, 8, and 9 immuno-fluorescent staining}

Approximately 1 or $2 \times 10^{5}$ cells (UMUC-3, WH, 253J, and BdSMC) were seeded on sterile coverslips in a 6-well plate overnight, either transduced by either rAd/eGFP or rAd/ dnStat 3 ( $\mathrm{moi}=500$ ) for 48 or 28 hours respectively, for UMUC-3 and WH cells. For the small molecule inhibition, cells were treated with $30 \mu \mathrm{M}$ STA-21 for 72 hours. Cleaved caspase immunostaining and documentation were described previously [50]. The primary rabbit antibodies were diluted with 1:100, 1:50, and 1:100 dilutions, respectively, for detecting cleaved-caspase-3 (Asp175), cleaved-caspase-8 (Asp374), or cleaved-caspase-9 (Asp330) (Cell Signaling Tech)

\section{Abbreviations}

BdSMC: bladder smooth muscle cell; DMSO: dimethyl sulfoxide; MTT: [3-(4, 5-dimethyl-2-thiazolyl)-2, 5-diphenyl-2H-tetrazolium bromide]; PIAS: protein inhibitors of activated STATs; SHP1/2: SH2-containing tyrosine phosphotases; Stat3: signal transducer and activator of transcription 3 .

\section{Competing interests}

The authors declare that they have no competing interests.

\section{Authors' contributions}

CLC participated in experiment designs, coordinated the experiments, contributed to the analysis and interpretation of data, and drafted the manuscript. LC and JK carried out the Adeno-viral dnStat3 and STA-21 experiments. BH participated in cell growth inhibition experiments. AL and $\mathrm{VH}$ carried out western blot analysis. CC, FCH and GC, carried out immunohistochemical staining of tissue microarray slides. JL conceived the ideas, coordinated the experiments and supervised on the data analyses, interpretation and the manuscript draft. All authors read and approved the final manuscript.

\section{Acknowledgements}

This work was supported in part by a start-up fund from the Center for Childhood Cancer, The Research Institute at Nationwide Children's Hospital, Department of Pediatrics at the Ohio State University, a NCl grant (ROI CA0967I4) and a Susan G. Koman Breast Cancer Foundation grant to J. Lin. Thanks to Jason Canner for carefully reading this manuscript and valuable comments.

\section{References}

I. Bowman T, Garcia R, Turkson J, Jove R: STATs in Oncogenesis. Oncogene 2000, 19:2474-2488.

2. Garcia R, Yu C, Hudnall A, Catlett R, Nelson K, Smithgall T, Fujita D, Ethier S, Jove R: Constitutive Activation of STAT 3 in Fibroblasts Transformed by Diverse Oncoproteins and in Breast Carcinoma Cells. Cell Growth Differ 1997, 8: 1267-1275.

3. Chen CL, Hsieh FC, Lin J: Systemic evaluation of total Stat3 and Stat3 tyrosine phosphorylation in normal human tissues. Exp Mol Pathol 2006, 80:295-305.

4. Kamimura D, Ishihara K, Hirano T: IL-6 signal transduction and its physiological roles: the signal orchestration model. Rev Physiol Biochem Pharmacol 2003, I 49: I-38.

5. Valentino L, Pierre J: JAK/STAT signal transduction: Regulators and implication in hematological malignancies. Biochem Pharmacol 2006, $71: 7 \mid 3-721$.

6. Bromberg J, Wrzeszczynska M, Devgan G, Zhao Y, Pestell R, Albanese C, Darnell JJ: Stat3 as an oncogene. Cell 1999, 98:295-303. 
7. Buettner R, Mora L, Jove R: Activated STAT signaling in human tumors provides novel molecular targets for therapeutic intervention. Clin Cancer Res 2002, 8:945-954.

8. Song $\mathrm{H}$, Wang $\mathrm{R}$, Wang $\mathrm{S}$, Lin J: A low-molecular-weight compound discovered through virtual database screening inhibits Stat3 function in breast cancer cells. Proc Natl Acad Sci USA 2005, 102:4700-4705.

9. Jing N, Tweardy DJ: Targeting Stat3 in cancer therapy. Anticancer Drugs 2005, 16:601-607.

10. Knowles MA: Molecular subtypes of bladder cancer: Jekyll and Hyde or chalk and cheese? Carcinogenesis 2006, 27:36I-373.

II. Sanchez-Carbayo M, Socci ND, Lozano J, Saint F, Cordon-Cardo C: Defining molecular profiles of poor outcome in patients with invasive bladder cancer using oligonucleotide microarrays. J Clin Oncol 2006, 24:778-789.

12. Zieger K, Dyrskjot L, Wiuf C, Jensen JL, Andersen CL, Jensen KM, Orntoft TF: Role of activating fibroblast growth factor receptor 3 mutations in the development of bladder tumors. Clin Cancer Res 2005, II:7709-77I9.

13. Salz W, Eisenberg D, Plescia J, Garlick DS, Weiss RM, Wu XR, Sun TT, Altieri DC: A survivin gene signature predicts aggressive tumor behavior. Cancer Res 2005, 65:353I-3534.

14. Schultz IJ, Kiemeney LA, Witjes JA, Schalken JA, Willems JL, Swinkels DW, de Kok JB: Survivin mRNA expression is elevated in malignant urothelial cell carcinomas and predicts time to recurrence. Anticancer Res 2003, 23:3327-333I.

15. Schaaf A, Sagi S, Langbein S, Trojan L, Alken P, Michel MS: Cytotoxicity of cisplatin in bladder cancer is significantly enhanced by application of bcl-2 antisense oligonucleotides. Urol Oncol 2004, 22: 188-192.

16. Xia G, Kumar SR, Stein JP, Singh J, Krasnoperov V, Zhu S, Hassanieh L, Smith DL, Buscarini M, Broek D, Quinn DI, Weaver FA, Gill PS: EphB4 receptor tyrosine kinase is expressed in bladder cancer and provides signals for cell survival. Oncogene 2006, 25:769-780.

17. Korkolopoulou P, Lazaris A, Konstantinidou AE, Kavantzas N, Patsouris E, Christodoulou P, Thomas-Tsagli E, Davaris P: Differential expression of bcl-2 family proteins in bladder carcinomas. Relationship with apoptotic rate and survival. Eur Urol 2002, 4I:274-283.

18. Swana HS, Grossman D, Anthony JN, Weiss RM, Altieri DC: Tumor content of the antiapoptosis molecule survivin and recurrence of bladder cancer. N Engl I Med 1999, 341:452-453.

19. Itoh M, Murata T, Suzuki T, Shindoh M, Nakajima K, Imai K, Yoshida $\mathrm{K}$ : Requirement of STAT3 activation for maximal collagenase-I (MMP-I) induction by epidermal growth factor and malignant characteristics in T24 bladder cancer cells. Oncogene 2006, 25: I I95-I204.

20. Chen C-L, Loy A, Cen L, Chan C, Hsieh F-C, Cheng G, Wu B, Qualman SJ, Kunisada K, Yamauchi-Takihara K, Lin J: Signal transducer and activator of transcription 3 is involved in cell growth and survival of human rhabdomyosarcoma and osteosarcoma cells. BMC Cancer 2007, 7: III.

21. Zhong Z, Wen Z, Darnell J: Stat3: a STAT family member activated by tyrosine phosphorylation in response to epidermal growth factor and interleukin-6. Science 1994, 264:95-98.

22. Darnell JJ, Kerr I, Stark G: Jak-STAT pathways and transcriptional activation in response to IFNs and other extracellular signaling proteins. Science 1994, 264:|4|15-|42I.

23. Kunisada K, Tone E, Fujio Y, Matsui H, Yamauchi-Takihara K, Kishimoto T: Activation of gp I 30 transduces hypertrophic signals via STAT3 in cardiac myocytes. Circulation 1998, 98:346-352.

24. Funamoto M, Fujio $Y$, Kunisada K, Negoro S, Tone E, Osugi T, Hirota $\mathrm{H}$, Izumi M, Yoshizaki K, Walsh K, Kishimoto T, Yamauchi-Takihara $\mathrm{K}$ : Signal transducer and activator of transcription 3 is required for glycoprotein 130 -mediated induction of vascular endothelial growth factor in cardiac myocytes. J Biol Chem 2000, 275: $1056 \mid-10566$

25. Hirano T, Ishihara K, Hibi M: Roles of STAT3 in mediating the cell growth, differentiation and survival signals relayed through the IL-6 family of cytokine receptors. Oncogene 2000, 19:2548-2556.

26. Levy DE, Darnell JE Jr: Stats: transcriptional control and biological impact. Nat Rev Mol Cell Biol 2002, 3:65I-662.

27. Takeda K, Noguchi K, Shi W, Tanaka T, Matsumoto M, Yoshida N, Kishimoto T, Akira S: Targeted disruption of the mouse Stat3 gene leads to early embryonic lethality. Proc Natl Acad Sci USA 1997, 94:380I-3804.

28. Amin HM, McDonnell TJ, Ma Y, Lin Q, Fujio Y, Kunisada K, Leventaki V, Das P, Rassidakis GZ, Cutler C, Medeiros LJ, Lai R: Selective inhibition of STAT3 induces apoptosis and $G(I)$ cell cycle arrest in ALK-positive anaplastic large cell lymphoma. Oncogene 2004, 23:5426-5434.

29. Cory S, Huang DC, Adams JM: The Bcl-2 family: roles in cell survival and oncogenesis. Oncogene 2003, 22:8590-8607.

30. Adams JM, Cory S: Apoptosomes: engines for caspase activation. Curr Opin Cell Biol 2002, I4:7I5-720.

31. Cory S, Adams JM: The Bcl2 family: regulators of the cellular life-or-death switch. Nat Rev Cancer 2002, 2:647-656.

32. Ashkenazi A, Dixit VM: Apoptosis control by death and decoy receptors. Curr Opin Cell Biol 1999, I I:255-260.

33. Ivanov VN, Bhoumik A, Ronai Z: Death receptors and melanoma resistance to apoptosis. Oncogene 2003, 22:3152-316I.

34. Shivapurkar N, Toyooka S, Toyooka KO, Reddy J, Miyajima K, Suzuki M, Shigematsu H, Takahashi T, Parikh G, Pass HI, Chaudhary PM, Gazdar AF: Aberrant methylation of trail decoy receptor genes is frequent in multiple tumor types. Int J Cancer 2004, 109:786-792.

35. Butcher BA, Kim L, Panopoulos AD, Watowich SS, Murray PJ, Denkers EY: IL-10-independent STAT3 activation by Toxoplasma gondii mediates suppression of IL- 12 and TNF-alpha in host macrophages. J Immunol 2005, I74:3 I48-3 I52.

36. Nishiki S, Hato F, Kamata N, Sakamoto E, Hasegawa T, Kimura-Eto A, Hino M, Kitagawa S: Selective activation of STAT3 in human monocytes stimulated by G-CSF: implication in inhibition of LPS-induced TNF-alpha production. Am J Physiol Cell Physiol 2004, 286:CI302-I3III.

37. Niu G, Shain KH, Huang M, Ravi R, Bedi A, Dalton WS, Jove R, Yu H: Overexpression of a dominant-negative signal transducer and activator of transcription 3 variant in tumor cells leads to production of soluble factors that induce apoptosis and cell cycle arrest. Cancer Res 200I, 6 I:3276-3280.

38. Lee Jl, Choi DY, Chung HS, Seo HG, Woo HJ, Choi BT, Choi YH: beta-lapachone induces growth inhibition and apoptosis in bladder cancer cells by modulation of Bcl-2 family and activation of caspases. Exp Oncol 2006, 28:30-35

39. Cho HJ, Kim JK, Kim KD, Yoon HK, Cho MY, Park YP, Jeon JH, Lee ES, Byun SS, Lim HM, Song EY, Lim JS, Yoon DY, Lee HG, Choe YK: Upregulation of $\mathrm{Bcl}-2$ is associated with cisplatin-resistance via inhibition of Bax translocation in human bladder cancer cells. Cancer Lett 2006, 237(I):56-66.

40. Calvin D, Nam S, Buettner R, Sekharam M, Torres-Roca J, Jove R: Inhibition of STAT3 activity with STAT3 antisense oligonucleotide (STAT3-ASO) enhances radiation-induced apoptosis in DUI45 prostate cancer cells. Int J Radiat Oncol Biol Phys 2003, 57:S297.

4I. Lee SO, Lou W, Qureshi KM, Mehraein-Ghomi F, Trump DL, Gao AC: RNA interference targeting Stat 3 inhibits growth and induces apoptosis of human prostate cancer cells. Prostate 2004, 60:303-309.

42. Turkson J, Zhang S, Mora LB, Burns A, Sebti S, Jove R: A novel platinum compound inhibits constitutive Stat 3 signaling and induces cell cycle arrest and apoptosis of malignant cells. $J$ Biol Chem 2005, 280:32979-32988.

43. Leong P, Andrews G, Johnson D, Dyer K, Xi S, Mai J, Robbins P, Gadiparthi S, Burke N, Watkins S, Grandis J: Targeted inhibition of Stat3 with a decoy oligonucleotide abrogates head and neck cancer cell growth. Proc Natl Acad Sci USA 2003, 100:41 38-4I 43.

44. Wu R, Sun S, Steinberg BM: Requirement of STAT3 activation for differentiation of mucosal stratified squamous epithelium. Mol Med 2003, 9:77-84.

45. Bhattacharya S, Ray RM, Johnson LR: STAT3-mediated transcription of Bcl-2, Mcl-I and c-IAP2 prevents apoptosis in polyamine-depleted cells. Biochem J 2005, 392:335-344.

46. Masuda M, Suzui M, Lim JT, Deguchi A, Soh JW, Weinstein IB: Epigallocatechin-3-gallate decreases VEGF production in head and neck and breast carcinoma cells by inhibiting EGFRrelated pathways of signal transduction. J Exp Ther Oncol 2002, 2:350-359.

47. Real PJ, Sierra A, De Juan A, Segovia JC, Lopez-Vega JM, FernandezLuna JL: Resistance to chemotherapy via Stat3-dependent 
overexpression of $\mathrm{Bcl}-2$ in metastatic breast cancer cells. Oncogene 2002, 21:761 I-7618.

48. Burke WM, Jin X, Lin HJ, Huang M, Liu R, Reynolds RK, Lin J: Inhibition of constitutively active Stat3 suppresses growth of human ovarian and breast cancer cells. Oncogene 200I, 20:7925-7934.

49. Hsieh FC, Cheng G, Lin J: Evaluation of potential Stat3-regulated genes in human breast cancer. Biochem Biophys Res Commun 2005, 335:292-299.

50. Chen C-L, Hsieh F-C, Brown J, Chan C, Wallaca JA, Cheng G, Hall $B M$, Lin J: Stat 3 activation in human endometrial and cervical cancers. Br J Cancer 2007, 96(4):59I-599.

Publish with Biomed Central and every scientist can read your work free of charge

"BioMed Central will be the most significant development for disseminating the results of biomedical research in our lifetime. "

Sir Paul Nurse, Cancer Research UK

Your research papers will be:

- available free of charge to the entire biomedical community

- peer reviewed and published immediately upon acceptance

- cited in PubMed and archived on PubMed Central

- yours - you keep the copyright

Submit your manuscript here:

http://www.biomedcentral.com/info/publishing_adv.asp
BioMedcentral 